\title{
MEMORIAS UNIVERSITARIAS
}

Publicamos aquí los títulos de las memorias universitarias que proporcionan una información que interesa a la geografía o que tocan incidentalmente temas geográficos, aunque no sea éste el aspecto principal de la memoria.

Es así como esta vez damos a conocer la lista previamente seleccionada de las memorias registradas en varias escuelas en el año 1964.

Todas las memorias enumeradas a continuación se pueden encontrar en las bibliotecas de las escuelas respectivas.

\section{INSTITUTO PEDAGOGICO DE LA UNIVERSIDAD CATOLIGA DE VALPARAISO}

(Nómina completa)

Valencia A., Nélida

Alberti B., Vito

Bianchetti V., Nenna

Salinas M., Rolando

Caviedes L., César
"La pesca en la zona de Valparaíso", 1958. 88 pp.

"El género Sardinops en el litoral norte de Chile", 1959. 90 pp., docs., planos.

"Esquema de la Geografía Urbana de Quillo ta", 1959. 88 pp.

"Ensayo de Geografía Urbana sobre San Francisco de Limache", 1959. 108 pp., planos.

"E1 puerto de Valparaíso", 1960. 120 pp. 
Gallardo O., Alicia

Gutiérrez T., Agtae

Guerrero P., Eriks

Núnez V., Luz

Vera H., Sergio

Vignal, Nilsa

Villalobos V., Ana

Cabrié, Aida

González, Rosa

Pizarro, Carlos
"La Cartografía nacional y los aportes de cuatro instituciones a su desarrollo", 1961. 133 PP.

"La prospección petrolifera en Tarapaca", 1961. $67 \mathrm{PP}$.

"Ensayo de Geografía Urbana de Quilpué", 1961. 127 pp., planos.

"El conocimiento de las costas chilenas a través de los descubrimientos y exploraciones de los siglos xvi y xvi", 1963. 257 pp., planos.

"Distribución del género Merluccius en el litoral de Chile", 1963. 180 pp.

"Diecisiete años de cabotaje 1946-1962. Un análisis del flujo por provincias", 1963. 74 PP., gráfs., apéndices.

"Las comunidades pesqueras de El Membrillo y Portales", 1965. 288 pp.

"E1 bosque relicto de Mantegua", $1965.76 \mathrm{Pp}$.

\section{ESCUELA DE AGRONOMIA DE LA UNIVERSIDAD DE CHILE} AÑ 1964

Gámez, Maria E.

Fernández, Miguel

Vender, Jorge

Maltrana, Antonio
"Factores de manejo que inciden en la producción lechera en la Hacienda "La Rinconada" de Maipú". 71 pp., gráfs.

"Comportamiento de trece variedades de sorgo (sorghum vulgare, Pres.) en el secano intetior (Maipú)" 76 pp., cuadros.

"Establecimiento de la mezcla Ballica Wim. mera (Lolium subulatum Vis.) y hualputra de California (Medicago htspida Gaertn) en condiciones de secano (Provincia de Santiago. Llano Central)". $90 \mathrm{pp}$., cuadros. 
Medina, Paulina

Morales, Héctor

Oliger, Leopoldo

Palacios, Alejandro

Pinto, Patricio

Ramírez, Manuel

Valjalo, Jorge
"Estudio microbiológico comparativo de la serie de suelos Lo Vásquez, bajo dos condiciones de manejo". 59 pp., figs., fotgs.

"Productividad presente y potencial en 96 predios de la provincia de O'Higgins y su relación con el tamaño de las propiedades". 113 pp., cuadros.

"La dosis más conveniente de fosfato para el establecimiento de una mezcla forrajera en nadis". 78 pp., fotgs.

"Estudio socioeconómico de la agricultura indigena en la provincia de Cautín". $309 \pm$ 27 pp., fotgs., cuadros.

"Determinación de uso, consumo y tasa de riego en malz de silo (Zea Mays L.) ". 65 pp., fotgs.

"Algunas caracteristicas lanimétricas de fibras de alpacas chilenas". 107 pp., fotgs., cuadros.

ESGUELA DE AGRONOMIA DE LA UNIVERSIDAD CATOLICA AÑO 1964

Arcaúz, Gastón

Campos, Carlos del

Cox, Tomás

Donoso, José
"Calibración de análisis de tierras, hojas y granos de trigo para el diagnóstico de fertilidad en suelos". 87 pp., fotgs., cuadros.

"Análisis de los efectos en Ia eficiencia agricola del sistema de inquilinaje y sistemas de pagos alternativos". $119 \mathrm{pp}$.

"Ensayo de fertilización en viñas de rulo en Portezuelo (Nuble)". 70 pp.

"Las plantaciones de limoneros en Chile y el control de sus heladas". 96 pp., figs., gráfs., cuadros. 
Downey, Ramón

Honorato, Ricardo

Matte, Jorge

Foch, Alberto

Irarrázaval, Rafael
"Una metodología simple, de análisis de eficiencia económica en la agricultura, en base a la función de producción. Provincia de O'Higgins". 58 pp., tablas.

"El análisis del grano de trigo, como método de diagnóstico de fertilidad de la tierra". $67 \mathrm{pp}$.

"Ensayo de rendimiento de 16 variedades de papa en contenido de almidón en la provincia de Valdivia, Valdivia". 71 pp., cuadros.

"Ensayos de abonos en praderas de alfalfa establecida. Valparaíso-Concepción". 68 pp., cuadros, tablas.

"Determinación de la unidad económica mediante programación lineal. Provincias de Santiago y O'Higgins". 111 pp., tablas.

\section{ESCUELA DE ARQUITECTURA DE LA UNIVERSIDAD DE CHILE}

\section{AÑO 1964.}

Balazs, León

De la Barra, Carlos

Concha, Raquel

Guzmán, Ana María

Fdding, Hernán.

Espina, Orlando

Véliz, Jaime

Hernández, Manuel

Poblete, Jaime

Lara, Gonzalo

Rendel, Gerardo
"Antecedentes urbanísticos para una planificación física educativa en la comuna de San Miguel". 90 pp., fotgs., planos., gráfs., cuadros.

"Análisis del equipamiento en poblaciones ubicadas en el bloque urbano S. O. de Santiago". 76 Pp., map., gráfs., planos, fotgs., figs.

"Estudio de un conjunto habitacional, población Exequiel González Cortés, "Villa Olímpica" ". 144 pp., cuadros, fotgs., planos.

“Análisis de la extensión urbana sur de Santiago metropolitano". 45 pp., gráfs., planos.

"Antecedentes para el desarrollo de comunidades mapuches en Cautín". 2223 pp., fotgs. 
Larsen, Federico

Salata, Orlando
"Expresión y medición del uso del suelo urbano". 174 pp., anexos, cuadros, maps., figs., fotgs.

“Análisis para un equipamiento rural Hacienda Mariposas. Talca". 108 pp., cuadros, maps., fotgs.

ESCUELA DE ARQUITECTURA DE LA UNIVERSIDAD CATOLICA ANTO 1964

Casanova, Juan

Ferrari, Claudio

García, José

Lohmann, Wolfgang

López, Hernando

Olavarría, Carlos

Purcell, Cedric

San Martín, Roberto

Urizar, Héctor

Irarrázaval, Raú!
"Centro turístico en Colchagua". Laminado.

"Remodelación de la Plazuela de Santa Ana y manzanas adyacentes". Laminado.

"Comunidad del mar". Laminado.

"Bodega, elaboración de vinos y organización generaI. Viña Guadalao". Laminado .

"Estación de ferrocarriles y terminal de turismo en Viña del Mar". Laminado.

"Centro industrial, agropecuario, conservero". Laminado.

"La comunidad del mar". Laminado.

"Organización de una parcela en el valle del Aconcagua". Laminado.

"Terminal pesquero en Angelmo". Laminado.

"Conjunto habitacional Santiago Poniente". Laminado.

ESCUELA DE DERECHO DE LA UNIVERSIDAD DE CHILE ANO 1964

Cabrera, Eduardo

"Reglamentación de comunidades". 124 pp.

Claro, Gustavo

"Derecho internacional de la propiedad industrial". $93 \mathrm{pp}$. 
Labra, Romilio

Salamero, Antonio

Santandreu, Ximena
"Constitución del dominio carbonifero". 89 pp.

"El régimen jurídico de la plataforma continental". 123 pp.

"Régimen jurídico de las substancias minera. les". $72 \mathrm{pp}$.

\section{ESCUELA DE DERECHO DE LA UNIVERSIDAD CATOLICA}

ANO 1964

Facusse, Francisco

Iturra, María

Sanfuentes, José

Sepúlveda, América

Undurraga, Claudio

Vial, Vicente

Zamora, Edgardo
"La fijación de los precios en el comercio interno de Chile". 192 pp.

"Legislación y política sobre turismo". 122 pp.

"Las pesquerfas nacionales y la legislación de formento". $170 \mathrm{pp}$.

"Legislación sobre reforma agraria". $67 \mathrm{pp}$.

"La expropiación de las tierras agricolas". $170 \mathrm{pp}$.

"El campesino chileno frente a la industria agrícola". $157 \mathrm{pp}$.

"E1 Instituto de Desarrollo Agropecuario". $48 \mathrm{pp}$.

\section{ESGUELA DE ECONOMIA DE LA UNIVERSIDAD DE CHILE} AÑO 1964

Alvarez, Patricio

Bifani, Pablo
"El problema de la mano de obra calificada y los centros de formación profesional y técnica en Chile". I55 pp., ilustrac.

"El mineral de fierro en Chile. Problemas de explotación y exportación". 243 pp., tablas. 
Donoso, Jaime

Escobar, Douglas

Guerra, Dilia

Guzmán, Sergio

Jadue, Santiago

Lazo, Mario

Mateluna, Sonia

Menéndez, Pedro

Mercado, Olga

Molina, Raúl

Morales, Rodolfo

Nahmlas, Abraham

Olavarria, Carlota
"Administración racional en la empresa agricola". 197 pp., ilustrac.

"Criterios de formación de áreas agricolas homogéneas y estimación de coeficientes de productividad. Estudio para la provincia de O'Higgins". s.n. tablas.

"EI problema habitacional de Arica y la urbanización". s.n.

"Aspectos tributarios de la industria extractiva del cobre en Chile". 61 pp., tablas.

"La madera aserrada en Chile, sus mercados internos y externos". $163 \mathrm{pp}$.

"La exportación chilena de cobre durante el período 1810-1910". s. n. tablas.

"Excedentes agrícolas". 123 pp., ilustrac.

"La mineria en Chile. Algunos antecedentes económicos". 168 pp., tablas.

"Política de integración socioeconómica de poblaciones marginales de los principales centros urbanos de Chile". s. n. tablas.

"Las poblaciones marginales en la provincia de Llanquihue. Aspectos urbano habitacional". 89 pp.

"Recursos humanos en la actividad pesque. ra". 123 pp., tablas.

"Indicadores de nivel de vida. Utilización de algunos de ellos en dos comunas de la provincia de Aconcagua". s. n. tablas,

"Perspectivas de la producción nacional de tres especies frutales $y$ análisis de su mercado". 44 pp., diags., tablas. 
Rapiman, Ninta

Sáenz, Juan

Soto, Sylvia
"Características de la distribución del ingre so en el Gran Santiago, y Concepción-Talcahuano". s. n. diags., tablas.

"El programa de créditos para mecanización agrícola de la OORFo, años 1960-1968'. 50 pp., ilustrac.

"Algunos aspectos de un plan de desarrollo económico para el departamento de Arica". 97 pp., ilustrac.

\section{ESCUELA DE ECONOMIA DE LA UNIVERSIDAD CATOLICA AÑO 1964}

Canales, Juan

Ossio, Hugo

Velasco, César
"Producción manufacturera y crédito". $64 \mathrm{pp}$

"El crédito agrícola supervisado en Chile". s. n. cuadros, diags.

"El vino chileno y la Asociación Latinoamericana de Libre Comercio". 177 pp., diags.

ESCUELA DE GEOLOGIA DE LA UNIVERSIDAD DE CHILE ANO 1964

Biró, Lajos

Cañas, Jorge

Hauser, Arturo

Lillo, Luisa
"Estudio sobre el límite entre el Titoniano y el Neocomiano en la Formación Lo Valdés, provincia de Santiago, principalmente en base a ammonoideos". 118 pp., láms., map., figs., planos.

"Tectónica y sedimentación de la Formación Los Molles, provincia de Aconcagua, Chile". 198 pp., fotgs., planos.

"La zona glauconf́tica en la plataforma Springhill, Magallanes, Chile”. 266 pp., láms., planos.

"Estudio geoquímico de las aguas del río Aconcagua". 135 pp., figs., fotgs., planos. 
Marangunic, Cedomir

Oyarzún, Jorge

Schultz, Augusto

“Observaciones glaciológicas y geológicas en la zona del Paso de los Cuatro Glaciares, Hielo Patagónico Sur". 125 pp., fotgs., figs., planos.

"Estudio de migración de cobre en el ciclo exógeno". 144 pp., map., fotgs., figs.

"Geología regional de Concepción". 116 pp., Jáms., gráfs., planos.

\section{ESCUELA DE SERVICIO SOCIAL DE LA UNIVERSIDAD DE CHILE}

ANO 1964

Aguirre, Nancy

Hernández, Edith

Fritz, Ruth

"Una experiencia sobre multiactividades en Cooperativas de Ahorro y Créditos urba. nos". 94 pp., anexos, cuadro.

Núñex, Adriana

"Ensayo de calificación de los niveles técnicos que han alcanzado trabajos de desarrollo de comuniclades en Santiago". 75 pp., cuadros, anexos.

Orozco, Alba

"Descripción antropológica de los pescadores de la caleta de San Antonio". 64 pp., fotgs., anexos.

\section{ESCUELA DE SERVICIO SOCIAL DE LA UNIVERSIDAD CATOLICA} ANO 1964

I.agos, Gloria

"Monografía de la provincia de O'Higgins". $86 \mathrm{pp}$., fot ${ }_{3}$., gráfs., maps.

\section{ESCUELA DE INGENIERIA DE LA UNIVERSIDAD DE CHILE ANO 1964}

Aros, Victor

"Proyecto de regadío del valle Nilahue". 254 pp., gráfs., planos.

Behn, Bruno

"Contribución al estudio y solución de problemas de ventilación de minas metálicas chilenas". 152 pp., ilustrac., fotgs., planos. 
Bezanilla, Fernando

Bustos, Maximiliano

Del Pino, Alvaro

Fotiu, Dimitrie

Guzmán, Fernando

Guzınán, Alfonso

Marín, Emilio

Morales, Jaime

Paz, Saúl

Rubio, Jorge

Schömherr, Jorge

Sotomayor, Raúl
"Programa de obras portuarias básicas entre Arica y Valparaiso, 1965-70". 332 pp., map., gráfs.

"Beneficios de los proyectos de regadio de Nilahue, Puclaro y Collipulli". 135 pp., tablas.

"Programa de obras portuarias básicas entre 1965-70. San Antonio-Punta Arenas". 1202 pp., ilustrac., planos, gráfs.

"Agua potable de Valparaiso. Captación de Las Vegas, descripción y análisis de los diferentes proyectos". 29 Pp., fotgs., planos.

"Estudio del riesgo sismico de la ciudad de Santiago". 64 pp., ilustrac.

"Proyecto general para un aeropuerto principal en Aisén". 185 pp., ilustrac., planos.

"Medida continua espectrofotométrica de ozono a muy baja concentración en la atmósfeta de Santiago". 79 pp., ilustrac.

"Anteproyecto de aeropuerto para Vallenar". 162 pp., ilustrac.

"Comparación de los costos marítimos y terres. tres de transporte de carga entre Chile y Argentina a través del Paso del Pehuenche para tres productos típicos de exportación". $106 \mathrm{pp}$., ilustrac.

"Planificación de una red de caminos en la provincia de Concepción al desarrollo". 142 pp. ilustrac., grafs., planos.

"Electrólisis del sulfato de cobre mediante baterías solares en la provincia de Antofagasta". 105 pp., ilustrac., gráfs.

"Estudio y prospección geofísica de Aguas subterráneas en la cuenca de Batuco". 104 pp., ilustrac. 
Ugarte, VIctor

Urrutia, Adrián

Vial, Eduardo

Welkner, Peter

Yáñez, Domingo
"Análisis de la estructura productiva de la provincia de Magallanes". 598 pp., gráfs., maps.

"Uso de las aguas servidas y de los desperdicios de las ciudades en la agricultura". 2 vols., ilustrac., planos.

"Anteproyecto de la central "Cabimbao". 463 pp., ilustrac., gráfs., maps.

"Estudio de la sismicidad en Chile y su aplicación al cálculo antisísmico". 71 pp., ilustrac.

"Desarrollo agtícola de la zona de riego DiguaPerquilauquén". 132 Pp., planos.

\section{ESCUELA DE INGENIERIA DE LA UNIVERSIDAD CATOLICA}

ANO 1964

Alzérreca, Germán

"Proyecto de planta de chancado en minas Navio, para empresas industriales El Melón. S.

Gualda, Fernando

Masjuán, Víctor

A.". 198 pp., figs., fotgs.

"Solución del transporte en las regiones servidas por los actuales ramales ferroviarios de Curicó a Licantén, Pua a Sierra Nevada y Cocule a Lago Ranco". 192 pp, anexo.

Luders, Carl

Vial, Felipe

“Asismicidad". 167 pp., figs.

Widoycovich, Pedro

"Inyecciones en las fundaciones de la Central Rapel". 136 pp., anexos.

\section{INSTITUTO PEDAGOGICO DE LA UNIVERSIDAD CATOLICA}

ANO 1964

Escudero, Josefina

Guerrero, Sergio

Moreno, Ibis

"Panorama económico de los puertos chilenos y sus instalaciones". $206 \mathrm{pp}$.

"Los transportes y las comunicaciones y sus proyecciones en el desarrollo nacional". 186 pp. 
Julic, Estrella

Lamperein, Sonia

"Crecimiento demográfico. Problema habitacional, Chile". $164 \mathrm{pp}$.

Miranda, Francisco

"Estudio geográfico de las Islas de Juan Fernández". 161 pp., ilustrac.

\section{INSTIIUTO PEDAGOGICO DE LA UNIVERSIDAD DE CHILE \\ ANO 1964}

Alamos, Ruth

Muñoz, Nora

"Monografia urbana de la ciudad de Rancagua". 99 pp., diags., fotgs., planos.

Fernández, Antonio

"La caleta y su gente; Tumbes (Fstudio etnográfico)". 62 pp., map.

Flores, Wladimir

Rivera, Pedro

"Coelemu; bases para un estudio regional". 101 pp., ilustrac.

Hernándex, María

"Aspecto de geografía de Cauquenes". s. n., ilustrac.

Inostroza, María

Ugarte, Sylvia

"Un rincón lejano de Chile ... la comunidad pascuense". 207 pp., fotgs., maps.

Peña, Orlando

"Hacia la delimitación del área regional de Valparaíso; antecedentes, perspectivas $y$ problemas". 113 pp., ilustrac.

Vera, Miguel

"Ancud; ensayo de geografía urbana". 262 pp., ilustrac., maps. 